\title{
Tratamento cirúrgico do ceratocisto odontogênico por meio de enucleação e osteotomia periférica: relato de caso
}

Surgical treatment of dontogenic ceratocyst by enucleation and peripheral osteotomy: case report Tratamiento quirúrgico de ceratocístas dontogénicos por enucleación y osteotomía periférica: reporte de caso

Diogo Henrique Rodrigues MARQUES ${ }^{1}$

Maylson Alves Nogueira BARROS ${ }^{1}$

Vitor Bruno TESLENCO ${ }^{1}$

Cláudio Marcio SANTANA JUNIOR ${ }^{1}$

Lucas Marques MEURER ${ }^{1}$

Ariane Fernanda VERLINGUE ${ }^{1}$

Guilherme Nucci dos REIS ${ }^{2}$

${ }^{I}$ Residente em Cirurgia e Traumatologia Bucomaxilofacial, Associação Beneficente da Santa Casa, 79002-251 Campo Grande - MS, Brasil

${ }^{2}$ Preceptor do programa de residência em Cirurgia e Traumatologia Bucomaxilofacial, Associação Beneficente da Santa Casa, 79002 -251 Campo Grande - MS, Brasil

\section{Resumo}

Introdução: Os ceratocistos odontogênicos (CCA) são considerados raros cistos de desenvolvimento, derivados dos remanescentes da lâmina dentária, com atividade intraóssea benigna, porém localmente invasivo e agressivo. $\mathrm{O}$ tratamento para o ceratocisto odongênico é variado, podendo-se encontrar modalidades tais como:enucleação, isolada ou associada a curetagem, com osteotomia periférica, aplicação da solução de Carnoy ou crioterapia, descompressão, marsupialização e ressecções. Objetivo: O presente trabalho tem como objetivo relatar um caso de ceratocisto odontogênico, onde foi escolhida abordagem conservadora por curetagem e osteotomia periférica. Relato de caso: Paciente de 68 anos, leucoderma, referiu ao exame clínico dor espontânea em região retromolar esquerda e parestesia em lábio inferior. A paciente foi submetida a biopsia por aspiração e excisional, após confirmação histopatológica foi proposto uma enucleação associada a osteotomia periférica sob anestesia geral. A paciente permanece em acompanhamento clínico e radiográfico, sem sinais de recidiva da lesão. Conclusão: Embora apresentem um comportamento agressivo, os ceratocistos odontogêncios podem ser tratados com segurança, de forma conservadora, por meio de enucleação seguida de osteotomia periférica com mínimo de morbidade.

Descritores: Osteotomia; Curetagem; Cistos Odontogênicos.

\section{Abstract}

Introduction: Odontogenic keratocysts (OKC) are considered rare developmental cysts, derived from the remnants of the dental lamina, with benign but locally invasive and aggressive intraosseous activity. Treatment for odongenic keratocyst is varied, and may include modalities such as enucleation, isolated or associated with curettage, with peripheral osteotomy, application of Carnoy's solution or cryotherapy, decompression, marsupialization and resections. Objective: This paper aims to report a case of odontogenic keratocyst, where a conservative approach for curettage and peripheral osteotomy was chosen. Case Report: A 68-year-old leukoderm patient referred to the clinical examination spontaneous pain in the left retromolar region and lower lip paresthesia. The patient underwent aspiration and excisional biopsy, and after histopathological confirmation, an enucleation associated with peripheral osteotomy under general anesthesia was proposed. The patient remains under clinical and radiographic follow-up, without signs of lesion recurrence. Conclusion: Although aggressive, odontogenic keratocysts can be safely and conservatively treated by enucleation followed by peripheral osteotomy with minimal morbidity.

Descriptores: Osteotomy; Curettage; Odontogenic Cysts.

\section{Resumen}

Introduccion: Los queratoquistes odontogénicos (CCA) se consideran quistes raros del desarrollo, derivados de los restos de la lámina dental, con actividad intraósea benigna pero localmente invasiva y agresiva. El tratamiento para el queratoquiste odongénico es variado y puede incluir modalidades como enucleación, aislado o asociado con legrado, con osteotomía periférica, aplicación de la solución de Carnoy o crioterapia, descompresión, marsupialización y resecciones. Objetivo: Este artículo tiene como objetivo informar un caso de queratoquiste odontogénico, donde se eligió un enfoque conservador para el legrado y la osteotomía periférica. Relato de caso: Un paciente con leucodermo de 68 años se refirió al examen clínico de dolor espontáneo en la región retromolar izquierda y parestesia de labio inferior. El paciente fue sometido a aspiración y biopsia por escisión, y después de la confirmación histopatológica, se propuso una enucleación asociada con osteotomía periférica bajo anestesia general. El paciente permanece bajo seguimiento clínico y radiográfico, sin signos de recurrencia de la lesión. Conclusión: Aunque agresivos, los queratoquistes odontogénicos pueden tratarse de forma segura y conservadora mediante enucleación seguida de osteotomía periférica con una morbilidad mínima.

Descriptores: Osteotomía; Legrado; Quistes Odontogénicos.

INTRODUÇÃO

Os ceratocistos odontogênicos (CCA) são considerados raros cistos de desenvolvimento, derivados dos remanescentes da lâmina dentária, com atividade intraóssea benigna, porém localmente invasivo e agressivo. De acordo com a última edição da Classificação dos Tumores de Cabeça e Pescoço da Organização Mundial de Saúde, de janeiro de 2017, o até então tumor odontogênico queratocístico foi reclassificado para cisto, devido à escassez de evidências científicas que sustentem a atividade neoplásica dos ceratocistos. Esta patologia representa cerca de $10 \%$ de todas as lesões císticas dos maxilares ${ }^{1,2,3}$. Os ceratocistos apresentam taxas de prevalência entre a segunda e terceira décadas de vida, com uma predileção pelo sexo masculino, sendo a região posterior da mandíbula a área mais acometida.
Embora possa apresentar um comportamento agressivo, na maioria das vezes, são lesões que se desenvolvem de forma assintomática, sendo detectadas em exames radiográficos de rotina. Tendem a se desenvolver no sentido anteroposterior dos espaços ósseos intramedulares, promovendo dessa forma, um mínimo de expansão óssea. Quando atingem grandes volumes, sintomas como dor e parestesia podem se fazer presentes. Radiograficamente se apresentam como imagens radiolúcidas uni ou multiloculares bem delimitadas com bordas escleróticas, podendo ou não estar associada a um dente incluso. Especificamente em relação a variante radiográfica unilocular, as características podem causar dúvidas quanto ao diagnóstico, sendo necessário a realização de diagnóstico diferencial para cisto dentígero, cisto periodontal lateral e cistos radiculares. Os 
deslocamentos dentários e reabsorções radiculares também podem estar presentes nos achados radiográficos, embora a reabsorção radicular seja um achado incomum nesse tipo de lesão ${ }^{1,3-5}$.

Quanto as características histopatológicas, verifica-se uma fina camada de tecido epitelial escamoso uniforme paraqueratinizado de superfície corrugada, composto de 5 a 10 camadas de células basais em paliçada, estando os núcleos dispostos de forma vertical ${ }^{1,4}$. O tratamento para o ceratocisto odongênico é variado, podendo-se encontrar modalidades tais como: enucleação, isolada ou associada a curetagem, com osteotomia periférica, aplicação da solução de Carnoy ou crioterapia, descompressão, marsupialização e ressecções ${ }^{1-5}$. O presente trabalho tem como objetivo relatar um caso de ceratocisto odontogênico, onde foi escolhida abordagem conservadora por curetagem e osteotomia periférica.

\section{CASO CLÍNICO}

Paciente do gênero feminino, 68 anos, edêntula total, foi encaminhada ao ambulatório de Cirurgia e Traumatologia Bucomaxilofacial, queixando-se de dor espontânea em região retromandibular esquerda e parestesia do lábio inferior. Durante anamnese referia ser hipertensa em tratamento, negava história de trauma na região. Ao exame clínico intraoral, a paciente apresentava-se com aumento de volume na região posterior de mandíbula esquerda. Ao exame radiográfico panorâmico solicitado, evidenciou-se uma imagem radiolúcida multilocular bem delimitada por margens escleróticas, localizada na região de corpo e ramo ascendente mandibular esquerdo (Figura 1).

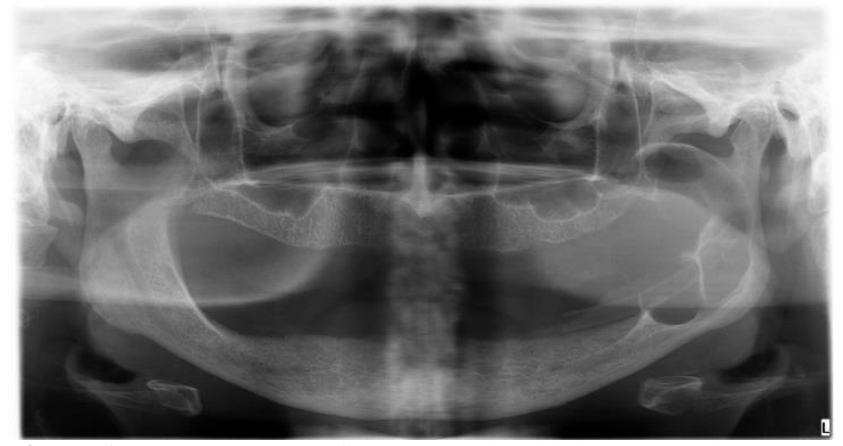

Figura 1: Imagem radiográfica panorâmica inicial, evidenciando uma lesão radiolúcida bem delimitada em corpo e ramo mandibular esquerdo.

A paciente foi submetida à biopsia por aspiração e excisional sob anestesia local e encaminhado o material para análise, com base no resultado do exame histopatológico, que evidenciou características peculiares da patologia tais como: fina camada de tecido epitelial escamoso uniforme paraqueratinizado de superfície corrugada, 5 a 10 camadas de células basais em paliçada ${ }^{1,4}$, o diagnóstico de ceratocisto odontogênico foi confirmado. Após confirmação histopatológica foi proposto uma enucleação associada a osteotomia periférica sob anestesia geral. A técnica cirúrgica realizada foi constituída de anestesia infiltrativa com lidocaína a 2\%, com adrenalina 1:100.000 para hemostasia local, incisão retromandibular, aproximadamente $0,5 \mathrm{~cm}$, com bisturi elétrico monopolar, descolamento mucoperiosteal e exposição da lesão. Então foi feito um acesso ósseo com broca esférica carbide e enucleação e curetagem do queratocisto associada a osteotomia periférica também com broca esférica carbide. Após remoção da lesão foi realizada sutura da mucosa com ácido poliglicólico 4.0. A paciente permanece em acompanhamento clínico e radiográfico até o momento, com total de 50 meses de acompanhamento, sem sinais de recidiva da lesão (Figuras 2 a 4).

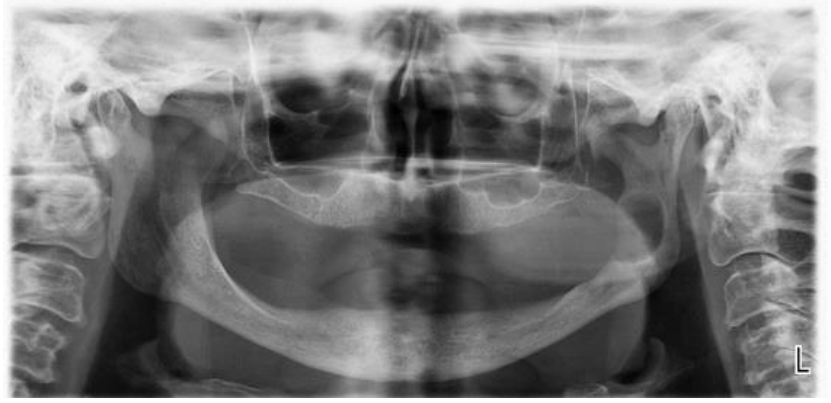

Figura 2: Imagem radiográfica após 1 ano da enucleação. Observa-se regressão da lesão.

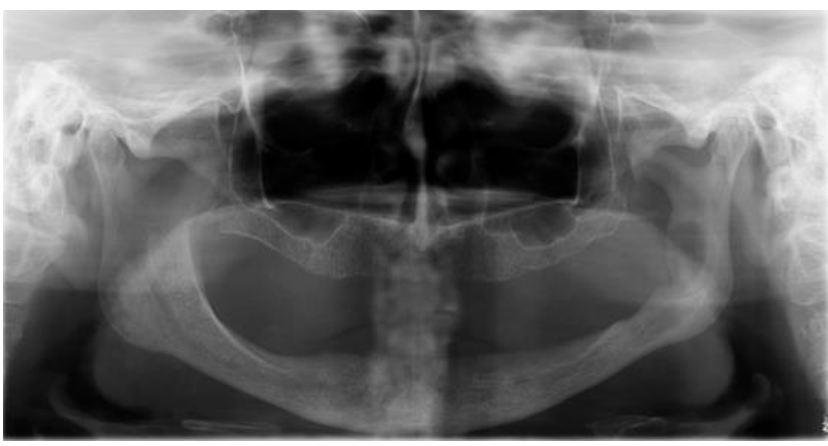

Figura 3: Imagem radiográfica após 3 anos da enucleação. Observa-se regressão da lesão.

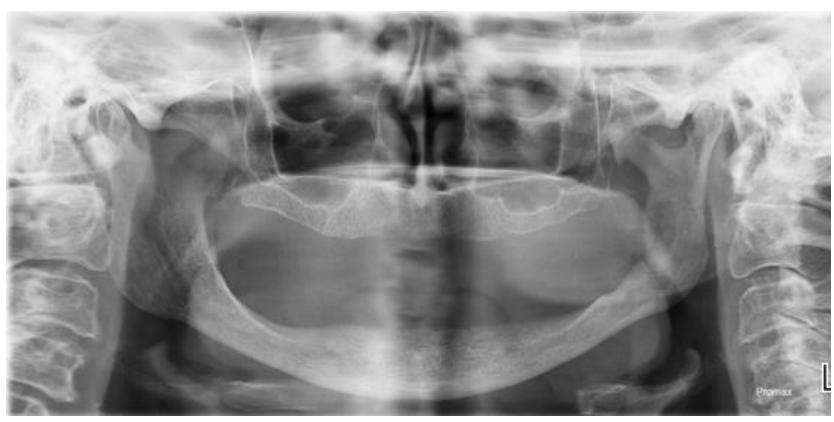

Figura 4: Imagem radiográfica após 50 meses da enucleação. Lesão regredindo sem sinal de recidiva.

DISCUSSÃO

Os cistos odontogênicos de desenvolvimento se formam a partir dos remanescentes epiteliais durante as diferentes fases da odontogênese. Segundo Borghesi et al. ${ }^{1}$ o ceratocisto apresenta uma taxa de prevalência de aproximadamente $10 \%$, enquanto Karaca et al. ${ }^{3}$ afirmam em seus estudos que a prevalência pode chegar a $21,8 \%$, sendo, portanto, 
um dos cistos odontogênicos mais presentes nos ossos maxilares. Para Freitas et al. ${ }^{6}$ o ceratocisto ao lado do cisto dentígero são os mais prevalentes. Park et $a^{2}{ }^{2}$ evidenciaram em seus estudos sobre o padrão de redução da descompressão no ceratocisto odontogênico em mandíbula, uma maior incidência da lesão entre a primeira e terceira décadas vida.

Borghesi et al. ${ }^{1}$, Freitas et al. $^{6}$ e Madhusudhan et al. ${ }^{7}$ afirmaram que a faixa etária prevalente dessa patologia é ampla, podendo surgir da primeira a nona década de vida, embora apresente um pico de incidência entre a segunda e terceira décadas de vida. De acordo com a maior parte da literatura a mandíbula é mais acometida que a maxila, sendo a região de corpo mandibular e ramo ascendente os acidentes anatômicos mais atingidos ${ }^{7,8}$. Segundo Borghesi et al. ${ }^{1}$, Park et al. ${ }^{2}$, Guerra et al. ${ }^{4} \mathrm{e}$ Freitas et al. ${ }^{6}$ os homens são mais acometidos que as mulheres, enquanto Madhusudhan et $\mathrm{al}^{7}$ apresentaram em seus estudos uma frequência maior no sexo feminino. O caso relatado nesse trabalho apresenta dados compatíveis com o que é descrito na maior parte da literatura no que se refere a faixa etária e localização da lesão, porém contraria a maioria dos estudos em relação ao gênero.

Varoli et al. ${ }^{9}$ citam em seu artigo um estudo retrospectivo de 10 anos, onde os autores concluíram que a expansão óssea tende a ser a manifestação clínica mais comum do ceratocisto, contrariando a maior parte dos estudos sobre o tema, que afirmam que o crescimento dessa lesão cística se dá através dos espaços intramedulares, portanto, ao longo do osso, promovendo discreta expansão óssea nos estágios iniciais da doença ${ }^{1,4}$. Moura et al. ${ }^{8}$ afirmam que a ausência de expansão é o principal auxílio para realizar um diagnóstico diferencial com outras lesões císticas e tumorais como, cisto dentígero, cisto radicular e ameloblastoma. Neste relato de caso a paciente apresentava discreto abaulamento ósseo em região posterior de mandíbula esquerda, sinal clínico compatível com o que é encontrado na literatura. Radiograficamente os ceratocistos se apresentam como imagens radiolúcidas uni ou multiloculares com margens bem definidas, limitadas por bordas escleróticas ${ }^{1,4,9,10}$. Embora as imagens radiológicas desempenhem um papel de destaque no diagnóstico de ceratocistos, Borghesi et al. ${ }^{1}$ destacam que essas não são características patognomônicas, principalmente em lesões pequenas uniloculares, podendo simular outras lesões odontogênicas e não odontogênicas, como cisto radicular, cisto periodontal lateral ou cisto do ducto nasopalatino. Concordando com grande parte dos estudos, Varoli et al. ${ }^{9}$ afirmam que o padrão unilocular predomina sobre o multilocular, sendo este mais comum na região posterior de mandíbula. O padrão radiográfico encontrado no presente estudo se mostrou multilocular com septo interno dentro da lesão, portanto, é um caso menos prevalente, levando em consideração o que afirmam grande parte dos estudos sobre o padrão radiográfico dessa patologia.

O manejo do paciente com ceratocisto odontogênico tem como objetivo reduzir o risco de recorrência da lesão, e ao mesmo tempo, minimizar a morbidade para o indivíduo. Até o momento, não existe consenso em relação a melhor forma de tratamento. Borghesi et al. ${ }^{1}$ descreveram que vários fatores interferem na forma de tratamento, desde localização e tamanho da lesão até comprometimento de tecidos moles e idade. Esses mesmos autores citaram em seu artigo uma revisão sistemática na qual se chegou à conclusão que a enucleação foi a modalidade de tratamento com a maior taxa de recorrência, cerca de $30 \%$ dos casos estudados, seguido da marsupialização isolada, com aproximadamente $18 \%$ de taxa de recorrência, sendo a enucleação associada a cauterização química com solução de Carnoy a modalidade de tratamento com menor taxa de recidiva, cerca de $8 \%$. Park et al. ${ }^{2}$ consideraram a descompressão seguida de enucleação como a modalidade de tratamento mais conservadora e efetiva em comparação com as demais formas de abordagem cirúrgica. Esses autores concluíram em seu artigo que a descompressão além de preservar estruturas nobres, diminui complicações funcionais e estéticas. Apesar de considerarem a taxa de recorrência baixa para esse tipo de tratamento, esses autores fazem uma alerta quanto à necessidade de acompanhamento periódico por meio de exames de imagens. Este mesmo estudo traz como novidade a afirmação de que uma lesão cística leva em média 270 dias para reduzir pela metade o seu volume descomprimido. Indo a favor do que se encontra na maior parte da literatura, Karaca et al. ${ }^{3}$ relataram que a ressecção marginal ou em bloco se apresenta como a modalidade de tratamento com menor taxa de recidiva, embora só seja recomendada para lesões grandes, com frequente recorrência em áreas anatômicas difíceis, uma vez que, associa-se a morbidade como assimetria facial e perda de continuidade mandibular. Karaca et al. ${ }^{3}$ realizaram um estudo retrospectivo onde avaliou-se a taxa de recorrência em pacientes diagnosticados com ceratocisto odontogênico utilizando apenas a enucleação seguida de osteotomia periférica como modalidade de tratamento. A taxa de recorrência da lesão no referido estudo foi de $14,8 \%$ em uma média de acompanhamento de 5 anos. Os autores concluíram que a enucleação com osteotomia periférica pode ser utilizada com segurança para o tratamento do ceratocisto odontogênico com mínima morbidade. Acreditam ainda que a taxa de recidiva pode ser reduzida por meio de exames radiográficos pré-operatórios cuidadosos, além da realização de uma técnica cirúrgica meticulosa.

$\mathrm{O}$ tratamento realizado no presente relato de 
caso foi o defendido por Karaca et al. ${ }^{3}$ com aplicação de enucleação e osteotomia periférica, procedimento realizado com o máximo de precisão cirúrgica. Foi levado em consideração, na escolha do método de tratamento empregado, a idade da paciente e a menor morbidade da técnica cirúrgica.

CONCLUSÃO

Embora apresentem um comportamento agressivo, os ceratocistos odontogêncios podem ser tratados com segurança, de forma conservadora, por meio de enucleação seguida de osteotomia periférica com mínimo de morbidade. Acredita-se que a taxa de recorrência da lesão possa ser reduzida por meio de exames de imagens pré-operatórios criteriosos, bem como através da realização de uma técnica cirúrgica precisa. Ainda se faz necessário um acompanhamento radiológico periódico para os pacientes tratados cirurgicamente, em especial nos primeiros 5 anos.

\section{REFERÊNCIAS}

1. Borghesi A, Nardi C, Giannitto C, Tironi A, Maroldi R, Di Bartolomeo F, Preda L. Odontogenic keratocyst: imaging features of a benign lesion with an aggressive behaviour. Insights Imaging. 2018 Oct;9(5):883-897.

2. Park JH, Kwak EJ, You KS, Jung YS, Jung HD. Volume change pattern of decompression of mandibular odontogenic keratocyst. Maxillofac Plast Reconstr Surg. 2019 Jan 7;41(1):2.

3. Karaca C, Dere KA, Er N, Aktas A, Tosun E, Koseoglu OT, Usubutun A. Recurrence rate of odontogenic keratocyst treated by enucleation and peripheral ostectomy: Retrospective case series with up to 12 years of follow-up. Med Oral Patol Oral Cir Bucal. 2018 Jul 1;23(4):e443-e448.

4. Guerra LAP, Silva PS, Dos Santos RLO, Silva AMF, Albuquerque DP. Tratamento conservador de múltiplos tumores odontogênicos ceratocístico em paciente não sindrômico. Rev cir traumatol. buco-maxilo-fac. 2013; 13(2):43-50.

5. Sundaragiri KS, Saxena S, Sankhla B, Bhargava A. Non syndromic synchronous multiple odontogenic keratocysts in a western Indian population: A series of four cases. J Clin Exp Dent. 2018;10(8):e831-6.

6. Freitas AD, Veloso DA, Santos ALF, Freitas VA. Maxillary odontogenic keratocyst: a clinical case report. RGO Rev Gaúch Odontol. 2015; 63(4):484-88.

7. Madhireddy MR, Prakash AJ, Mahanthi V, Chalapathi KV. Large Follicular Odontogenic Keratocyst affecting Maxillary Sinus mimicking Dentigerous Cyst in an 8-year-old Boy: A Case Report and Review. Int J Clin Pediatr Dent. 2018 Jul-Aug;11(4):349-351.

8. Moura BS, Cavalcante MA, Hespanhol W. Tumor odontogênico ceratocistico. Rev Col Bras Cir., 2016;43(6):466-71.
9. Valori FP, Costa E, Buscatti MY, Oliveira JX, Costa C. Tumor odontogênico queratocístico: características intrínsecas e elucidação da nova nomenclatura do queratocisto odontogênico. J Health Sci Inst. 2010;28(1):80-3.

10. Slusarenko da Silva Y, Stoelinga PJW, NaclérioHomem MDG. The presentation of odontogenic keratocysts in the jaws with an emphasis on the tooth-bearing area: a systematic review and metaanalysis. Oral Maxillofac Surg. 2019;23(2):133-47.

\section{CONFLITO DE INTERESSES}

Os autores declaram não haver conflitos de interesse.

\section{AUTOR PARA CORRESPONDÊNCIA}

Diogo Henrique Rodrigues Marques

Rua Hermelita de Oliveira Gomes, 225 - Santa Fé. Cep: 79021-270. Campo Grande - MS, Brasil

E.mail: diogo.hrm@hotmail.com 